\title{
Case Report: SARS-CoV-2 as an unexpected causal agent of
}

\section{isolated febrile hepatitis [version 1; peer review: 1 approved}

\section{with reservations]}

\author{
Paraskevas Filippidis (iD)1, Francois van Ouwenaller², Alberto Cerutti (D)2, \\ Anaïs Geiger-Jacquod2,3, Christine Sempoux ${ }^{4}$, Giuseppe Pantaleo5,6, \\ Darius Moradpour ${ }^{3}$, Frederic Lamoth ${ }^{1,7}$
}

\begin{abstract}
${ }^{1}$ Infectious Diseases Service, Lausanne University Hospital and University of Lausanne, Lausanne, Vaud, 1011, Switzerland
2Internal Medicine Service, Lausanne University Hospital and University of Lausanne, Lausanne, Vaud, 1011, Switzerland

${ }^{3}$ Service of Gastroenterology and Hepatology, Lausanne University Hospital and University of Lausanne, Lausanne, Vaud, 1011, Switzerland

${ }^{4}$ Service of Clinical Pathology, Institute of Pathology, Lausanne University Hospital and University of Lausanne, Lausanne, Vaud, 1011, Switzerland

${ }^{5}$ Service of Immunology and Allergy, Department of Medicine, Lausanne University Hospital and University of Lausanne, Lausanne, Vaud, 1011, Switzerland

${ }^{6}$ Swiss Vaccine Research Institute, Lausanne University Hospital and University of Lausanne, Lausanne, Vaud, 1011, Switzerland

${ }^{7}$ Institute of Microbiology, Lausanne University Hospital and University of Lausanne, Lausanne, Vaud, 1011, Switzerland
\end{abstract}

\section{V1 First published: 18 May 2021, 10:400 \\ https://doi.org/10.12688/f1000research.52929.1}

Latest published: $07 \mathrm{Jul} 2021,10: 400$

https://doi.org/10.12688/f1000research.52929.2

\section{Abstract}

Background: Respiratory symptoms and pneumonia are the predominant features of Coronavirus disease 2019 (COVID-19) due to emerging SARS-CoV-2 virus, but extrapulmonary manifestations are also observed. For instance, some degree of liver injury has been described among patients requiring hospital admission for severe COVID-19. However, acute febrile hepatitis as an initial or predominant manifestation of COVID-19 has been rarely reported. Case presentation: A 34-year-old man without underlying medical conditions presented with fever of unknown origin for two weeks in the absence of respiratory symptoms or other complaints. Laboratory testing revealed isolated acute hepatitis, for which an extensive microbiological work-up did not reveal identification of the causal agent. PCR testing for SARS-CoV-2 on a nasopharyngeal swab was negative on two occasions and initial serology for SARS-CoV-2 (at 15 days from symptoms onset) was also negative. However, repeated SARS-CoV-2 serological testing at 30 days demonstrated seroconversion leading to the diagnosis of COVID-19-related hepatitis. The patient's condition progressively improved, while transaminases steadily declined and eventually returned back to normal within 30 days.

Conclusions: We describe here a unique case of SARS-CoV-2 isolated

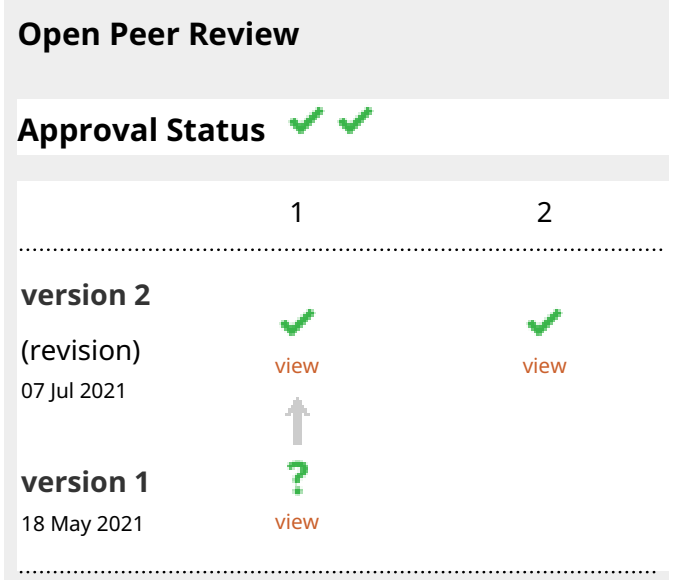

1. Werner C Albrich ID, Kantonsspital St. Gallen, St. Gallen, Switzerland

2. Marco Merli ID, ASST Grande Ospedale Metropolitano Niguarda, Milan, Italy

Any reports and responses or comments on the article can be found at the end of the article. 
febrile hepatitis in a young and previously healthy man, which was diagnosed by demonstration of seroconversion, while PCR screening was negative. This case report highlights the role of repeated serological testing for the diagnosis of extrapulmonary manifestations of COVID-19.

Keywords

COVID-19, hepatology, liver, serology

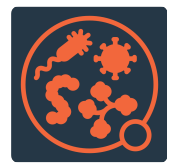

This article is included in the Pathogens

gateway.

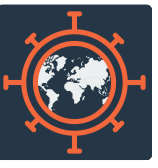

This article is included in the Emerging Diseases

and Outbreaks gateway.

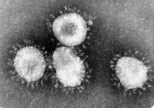

This article is included in the Coronavirus

collection.

\section{Corresponding author: Frederic Lamoth (Frederic.Lamoth@chuv.ch)}

Author roles: Filippidis P: Conceptualization, Data Curation, Resources, Visualization, Writing - Original Draft Preparation, Writing Review \& Editing; van Ouwenaller F: Writing - Review \& Editing; Cerutti A: Writing - Review \& Editing; Geiger-Jacquod A: Writing Review \& Editing; Sempoux C: Resources, Visualization, Writing - Review \& Editing; Pantaleo G: Writing - Review \& Editing; Moradpour D: Writing - Review \& Editing; Lamoth F: Conceptualization, Data Curation, Project Administration, Resources, Supervision, Writing Review \& Editing

Competing interests: No competing interests were disclosed.

Grant information: The author(s) declared that no grants were involved in supporting this work.

Copyright: $\odot 2021$ Filippidis P et al. This is an open access article distributed under the terms of the Creative Commons Attribution License, which permits unrestricted use, distribution, and reproduction in any medium, provided the original work is properly cited.

How to cite this article: Filippidis P, van Ouwenaller F, Cerutti A et al. Case Report: SARS-CoV-2 as an unexpected causal agent of isolated febrile hepatitis [version 1; peer review: 1 approved with reservations] F1000Research 2021, 10:400

https://doi.org/10.12688/f1000research.52929.1

First published: 18 May 2021, 10:400 https://doi.org/10.12688/f1000research.52929.1 


\section{Background}

Severe acute respiratory syndrome Coronavirus 2 (SARS-CoV-2), the etiological agent of Coronavirus disease 2019 (COVID-19) can cause a wide spectrum of clinical presentations ranging from mild flu-like illness to severe pneumonia with acute respiratory distress syndrome ${ }^{1}$. While the pandemic is ongoing, atypical presentations with extrapulmonary organ involvement, including heart, kidneys, skin, nervous system, hepatobiliary and gastrointestinal tract are increasingly recognized ${ }^{2}$. Liver injury with mild or moderate elevation of transaminases has been observed in about half of patients requiring hospital admission for severe COVID-193,4. However, acute febrile hepatitis as a predominant manifestation of COVID-19 has been rarely reported and has been mainly observed among patients with underlying liver diseases and/or concomitant signs of upper or lower respiratory tract involvement with diagnosis established by polymerase chain reaction (PCR) in nasopharyngeal swabs ${ }^{5-7}$. We report here a case of a young previously healthy man presenting with febrile hepatitis as a unique clinical manifestation, for which diagnosis of COVID-19 was established by seroconversion.

\section{Case presentation}

A 34-year-old previously healthy Caucasian man was admitted to our tertiary care hospital for investigation of fever of unknown origin. He had presented night fever up to $39.5^{\circ} \mathrm{C}$ with rare chills, night sweats and moderate headache for two weeks prior to admission. His symptoms responded to acetaminophen and nonsteroidal anti-inflammatory drugs. During the first week after fever onset, he had also presented with a mild sore throat and dry cough, which had completely resolved at the time of admission. Exposure history was remarkable with travel to a Greek island one month ago and a superficial scratch from his neighbor's cat two months ago. Upon admission, his vital signs and physical examination were unremarkable. Laboratory tests showed systemic inflammation with leukocytosis $(10.5 \mathrm{G} / \mathrm{l})$, thrombocytosis $(501 \mathrm{G} / \mathrm{l})$ and elevated inflammatory markers (C-reactive protein 126 $\mathrm{mg} / \mathrm{l}$, ferritin $695 \mu \mathrm{g} / \mathrm{l}$ ), as well as acute hepatitis with a threefold increase of transaminases, a two-fold increase of alkaline phosphatase and a four-fold increase of gamma-glutamyltranspeptidase. Total bilirubin was normal. Cervical, thoracic and abdominal computed tomography was unremarkable. PCR for SARS-CoV-2 on a nasopharyngeal swab was negative on two occasions. Serology for SARS-CoV-2 performed at admission (i.e. at 15 days from fever onset) was negative using the Luminex $\mathrm{S}$ protein trimer IgG assay, as previously described ${ }^{8}$. Serologies for hepatitis A, B, C and E viruses, Epstein-Barr virus, cytomegalovirus and human immunodeficiency virus were negative. Further microbiological diagnostic work-up, including serological testing for bartonellosis, Q fever, brucellosis, tularemia, Lyme disease, rickettsial diseases, toxoplasmosis, syphilis and leptospirosis was negative. Autoimmune and metabolic causes of hepatitis were also excluded. We consequently performed a percutaneous liver biopsy, which showed nonspecific acute lobular hepatitis with negative immunohistochemical staining for herpes viruses (Figure 1). Cultures of the liver tissue were sterile and broad-spectrum bacterial (16S

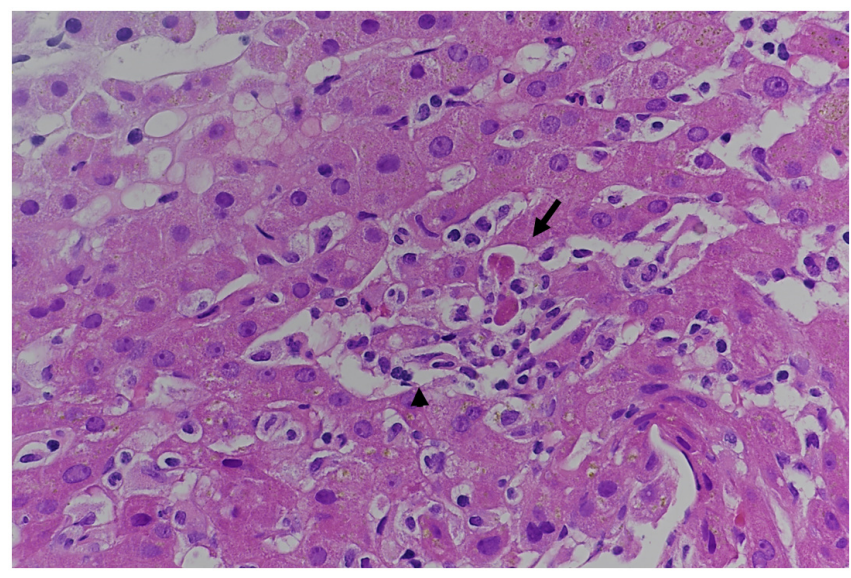

Figure 1. Histologic findings in liver biopsy. Mild lobular hepatitis with few apoptotic bodies (arrow) surrounded by mononuclear inflammatory cells (arrowhead). Hematoxylin-eosin staining, original magnification $\times 400$.

rDNA), fungal (18S rDNA) and mycobacterial PCRs, as well as specific PCRs for Brucella spp., Bartonella spp., Coxiella burnetii, were all negative. Transthoracic echocardiography found no endocarditis-related abnormality. Positron emission tomography (PET) revealed moderate hypermetabolism of the posterior naso-oropharynx and numerous hypermetabolic cervical lymph nodes. However, naso-oropharyngeal endoscopy and cervico-facial magnetic resonance imaging found no structural abnormality. Serologies for hepatitis A, B, C and E viruses, cytomegalovirus and Epstein-Barr virus were tested again at a two-week interval and remained negative. However, repeated SARS-CoV-2 serology (i.e. at 30 days from the onset of fever) turned out clearly positive. This result was confirmed on a subsequent serum sample collected one week later and showing increasing titers. Retrospective SARS-CoV-2 PCR testing in serum and in liver tissue was negative. As illustrated in Figure 2, the patient's condition progressively improved despite persistent low-grade fever at discharge, while transaminases steadily declined and eventually returned back to normal within 30 days. Fever had completely resolved after six weeks of follow-up.

\section{Discussion and conclusions}

Investigations of acute hepatitis includes a diagnostic work-up for classical hepatotropic viruses and some other well known, albeit rarer, infectious agents causing liver injury. We report here a clinical observation raising attention to a novel pathogen that should be considered and actively searched for in such situations: the emerging SARS-CoV-2 virus.

While concomitant liver injury has been commonly observed among patients with severe COVID-19 pneumonia ${ }^{3,4}$, clinical presentation of COVID-19 with hepatitis as the predominant feature has been rarely reported ${ }^{5-7}$. All these cases had positive PCR for SARS-CoV-2 in nasopharyngeal swabs at admission, three had concomitant pulmonary involvement and 


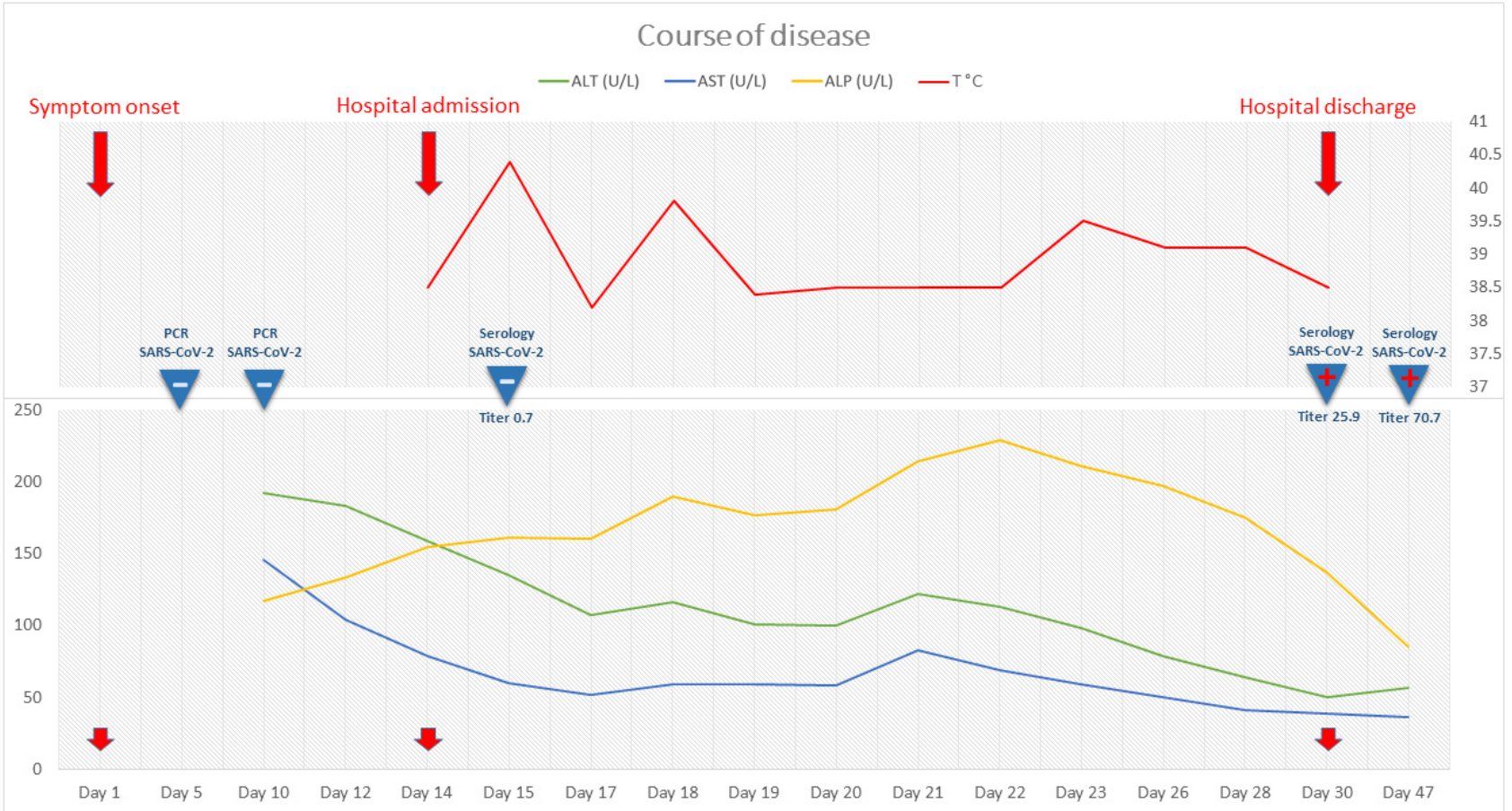

Figure 2. Clinical course of the disease for the present case report. Evolution of fever and liver function tests as well as results of SARS-CoV-2 PCR and serology. Serology testing for SARS-CoV-2 was performed by the Luminex S protein trimer IgG assay, as previously described $^{8}$. Results are expressed in mean fluorescence intensity ratio with a positive cut-off of 6 . ALT, alanine aminotransferase; AST, aspartate aminotransferase; ALP, alkaline phosphatase; $\gamma$-GT, $\gamma$-glutamyltranspeptidase.

two had predisposing liver conditions (e.g. chronic hepatitis $\mathrm{C}$ or previous liver transplantation).

Unlike previous reports ${ }^{5,7}$, our patient had no underlying liver disease and presented isolated fever and hepatitis with no radiological evidence of pulmonary involvement and negative SARS-CoV-2 PCR in nasopharyngeal swabs. Seroconversion occurring in a timeframe that was consistent with the course of the disease, after exclusion of other infectious and non-infectious causes of hepatitis, led to the diagnosis of COVID-19-related hepatitis in this case.

Interestingly, seroconversion occurred relatively late (between two and four weeks from fever onset). In a recent population-based seroprevalence study, the serological method used in this case (i.e. Luminex $\mathrm{S}$ protein trimer $\mathrm{IgG}$ assay) showed a sensitivity of $97 \%$ and a specificity equal or above $97 \%$, when performed by day 15 from symptom onset ${ }^{8}$. However, modest or delayed antibody responses (up to day 20) have been reported ${ }^{9}$, which could be more frequently observed in young patients with less severe forms of the disease. While specific data about the antibody response in patients with extrapulmonary manifestations of COVID-19 are lacking, the present case report highlights the role of serology for the diagnosis of these atypical forms presenting later in the course of the disease, when respiratory symptoms are absent or not predominant, and the need for repeated serological testing (up to 3-4 weeks from symptoms onset) in case of high clinical suspicion and/or absence of alternative diagnosis.

In the present case, attempts to demonstrate the presence of the virus in blood or liver tissue by PCR were unsuccessful. Indeed, the rate of positive SARS-CoV-2 PCR in non-respiratory samples, such as blood or deep-organ tissues, is notoriously low ${ }^{10}$. Although the nature of liver injury in COVID-19 remains to be elucidated, abnormal inflammatory response, rather than direct viral cytotoxicity, is suggested as the main pathophysiological mechanism of hepatitis ${ }^{11}$. A case series of patients deceased from COVID-19 showed lobular hepatitis in $50 \%$ of liver autopsies, but found no correlation between histologic findings and a positive PCR assay for SARS-CoV-2 on liver tissue ${ }^{12}$.

In conclusion, we report a case of SARS-CoV-2 infection with acute febrile hepatitis as a predominant manifestation. Importantly, this case highlights the need to include COVID-19 in the differential diagnosis of primary hepatitis while the pandemic is ongoing, and the crucial role of repeated serological testing (up to three to four weeks from symptoms onset) for the identification of such atypical extrapulmonary manifestations of the disease.

\section{List of abbreviations}

SARS-CoV-2 Severe acute respiratory syndrome 
COVID-19

Coronavirus disease 2019

PCR

Polymerase chain reaction

\section{Consent}

Written informed consent for publication of their clinical details and/or clinical images was obtained from the patient.

\section{Data availability}

All data underlying the results are available as part of the article and no additional source data are required.

Author contributions

P. Filippidis: clinical management of the case, data collection, figure design and drafting of manuscript.
F. Van Ouwenaller, A. Cerruti, A. Geiger-Jacquod: clinical management of the case, review and editing of manuscript.

C. Sempoux: interpretation of histopathology, figure design, review and editing of manuscript.

G. Pantaleo: interpretation of serological testing, review and editing of manuscript.

D. Moradpour: clinical management of the case, review and editing of manuscript

F. Lamoth: clinical management of the case, study design and writing of manuscript.

Acknowledgements

Not applicable.
1. Regina J, Papadimitriou-Olivgeris M, Burger R, et al:: Epidemiology, risk factors and clinical course of SARS-CoV-2 infected patients in a Swiss university hospital: An observational retrospective study. PLoS One. 2020; 15(11): e0240781.

PubMed Abstract | Publisher Full Text | Free Full Text

2. Wu Z, McGoogan JM: Characteristics of and Important Lessons From the Coronavirus Disease 2019 (COVID-19) Outbreak in China: Summary of a Report of 72314 Cases From the Chinese Center for Disease Control and Prevention. JAMA. 2020; 323(13): 1239-42.

PubMed Abstract | Publisher Full Text

3. Hao SR, Zhang SY, Lian JS, et al.: Liver Enzyme Elevation in Coronavirus Disease 2019: A Multicenter, Retrospective, Cross-Sectional Study. Am J Gastroenterol. 2020; 115(7): 1075-83.

PubMed Abstract | Publisher Full Text | Free Full Text

4. Zhang C, Shi L, Wang FS: Liver injury in CovID-19: management and challenges. Lancet Gastroenterol Hepatol. 2020; 5(5): 428-30. PubMed Abstract | Publisher Full Text | Free Full Text

5. Fiel MI, El Jamal SM, Paniz-Mondolfi A, et al.: Findings of Hepatic Severe Acute Respiratory Syndrome Coronavirus-2 Infection. Cell Mol Gastroentero Hepatol. 2021; 11(3): 763-770.

PubMed Abstract | Publisher Full Text | Free Full Text

6. Wander P, Epstein M, Bernstein D: COVID-19 Presenting as Acute Hepatitis.
Am J Gastroenterol. 2020: 115(6): 941-2.

PubMed Abstract | Publisher Full Text | Free Full Text

7. Trevenzoli M, Guarnaccia A, Alberici I, et al.: SARS-CoV-2 and hepatitis. J Gastrointestin Liver Dis. 2020; 29(3): 473-5.

PubMed Abstract | Publisher Full Text

8. Fenwick C, Croxatto A, Coste AT, et al.: Changes in SARS-CoV-2 Spike versus Nucleoprotein Antibody Responses Impact the Estimates of Infections in Population-Based Seroprevalence Studies. J Virol. 2021; 95(3): e01828-20. PubMed Abstract | Publisher Full Text | Free Full Text

9. $\quad$ Long QX, Liu BZ, Deng HJ, et al:: Antibody responses to SARS-CoV-2 in patients with COVID-19. Nat Med. 2020; 26(6): 845-8. PubMed Abstract | Publisher Full Text

10. Wang W, Xu Y, Gao R, et al.: Detection of SARS-CoV-2 in Different Types of Clinical Specimens. JAMA. 2020; 323(18): 1843-4. PubMed Abstract | Publisher Full Text | Free Full Text

11. Li J, Fan JG: Characteristics and Mechanism of Liver Injury in 2019 Coronavirus Disease. J Clin Transl Hepatol. 2020; 8(1): 13-7. PubMed Abstract | Publisher Full Text | Free Full Text

12. Lagana SM, Kudose S, Iuga AC, et al.: Hepatic pathology in patients dying of COVID-19: a series of $\mathbf{4 0}$ cases including clinical, histologic, and virologic data. Mod Pathol. 2020; 33(11): 2147-55. PubMed Abstract | Publisher Full Text | Free Full Text 


\section{Open Peer Review}

\section{Current Peer Review Status: ?}

\section{Version 1}

Reviewer Report 28 June 2021

https://doi.org/10.5256/f1000research.56261.r85690

(C) 2021 Albrich W. This is an open access peer review report distributed under the terms of the Creative Commons Attribution License, which permits unrestricted use, distribution, and reproduction in any medium, provided the original work is properly cited.

\section{Werner C Albrich}

Division of Infectious Diseases and Hospital Epidemiology, Kantonsspital St. Gallen, St. Gallen, Switzerland

The authors describe the rare case of a 34 yo male with acute Covid-19 infection which manifested mainly as acute and self-limiting hepatitis aside from mild upper respiratory symptoms, treated with acetaminophen and NSAIDs. Despite nasopharyngeal testing with PCR and liver biopsy for SARS-CoV-2 PCR, only seroconversion for SARS-CoV-2 was positive. Extensive work-up of alternative causes of the acute hepatitis were all negative.

\section{Minor comments:}

Should provide more detail on doses and frequency of acetaminophen and NSAIDs used for his hepatitis.

Did he take any herbal medications for the cold symptoms?

Provide more detail of tests for autoimmune and metabolic causes of the hepatitis.

Was there any pathological evidence of endotheliitis on liver biopsy?

Generally speaking, I agree with the authors' conclusions but would rephrase the title and manuscript text in that the hepatitis was not isolated but predominant.

Is the background of the case's history and progression described in sufficient detail? Partly

Are enough details provided of any physical examination and diagnostic tests, treatment given and outcomes?

Partly 
Is sufficient discussion included of the importance of the findings and their relevance to future understanding of disease processes, diagnosis or treatment?

Yes

Is the case presented with sufficient detail to be useful for other practitioners?

Yes

Competing Interests: No competing interests were disclosed.

Reviewer Expertise: Infectious diseases

I confirm that I have read this submission and believe that I have an appropriate level of expertise to confirm that it is of an acceptable scientific standard, however I have significant reservations, as outlined above.

Author Response 30 Jun 2021

Paraskevas Filippidis, Lausanne University Hospital and University of Lausanne, Lausanne, Switzerland

Dear reviewer,

Thank you for your precious feedback about our article and for the questions you submitted. Please find below point-to-point answers to your questions.

Should provide more detail on doses and frequency of acetaminophen and NSAIDs used for his hepatitis.

Response: Acetaminophen was taken at a dose of $500 \mathrm{mg}$ once or twice per day on demand during 14 days, namely a cumulative dose of $13.5 \mathrm{~g}$ upon admission, while the nonsteroidal anti-inflammatory drug nimesulid was taken at a dose of $100 \mathrm{mg}$ once per day on demand during 14 days, namely a cumulative dose of $900 \mathrm{mg}$ upon admission. In fact, these medications were rather used for the febrile syndrome than for his hepatitis and doses were relatively low to explain the liver damage observed. In addition, liver enzymes progressively normalized despite the continuous administration of the above mentioned medication to treat fever.

Did he take any herbal medications for the cold symptoms?

Response: No herbal medications or dietary supplements were taken by the patient.

Provide more detail of tests for autoimmune and metabolic causes of the hepatitis.

Response: Autoimmune and metabolic diagnostic work-up included antinuclear antibodies, rheumatoid factor, complement, total immunoglobulin G, complete autoantibody panels for autoimmune hepatitis and vasculitis, copper and ceruloplasmin values and normal thyroid tests, which were all normal. Liver imaging was also normal.

Was there any pathological evidence of endotheliitis on liver biopsy?

Response: No pathological evidence of endotheliitis was found in liver biopsy. 
Generally speaking, I agree with the authors' conclusions but would rephrase the title and manuscript text in that the hepatitis was not isolated but predominant.

Response: We rephrased the title's manuscript as to indicate a predominant rather than isolated character of patient's febrile hepatitis.

On behalf of my co-authors,

Paraskevas Filippidis

Competing Interests: No competing interests were disclosed.

The benefits of publishing with F1000Research:

- Your article is published within days, with no editorial bias

- You can publish traditional articles, null/negative results, case reports, data notes and more

- The peer review process is transparent and collaborative

- Your article is indexed in PubMed after passing peer review

- Dedicated customer support at every stage

For pre-submission enquiries, contact research@f1000.com 